\title{
L'électronucléaire à l'heure de l'Europe
}

\author{
Présentation \\ par Bernard DUPRAZ, Président de la SFEN
}

Dans ce traditionnel bilan que la RGN propose chaque année à ses lecteurs, le point commun des principaux domaines concernant l'électronucléaire est sans conteste l'Europe.

Alors que les programmes nucléaires «des trente glorieuses" correspondaient à des logiques nationales, de politiques énergétique et industrielle, l'année écoulée a vu se renforcer considérablement la dimension européenne de l'énergie nucléaire, qui avait commencé à se développer au début des années 1990, et ce aux plans politique, énergétique, environnemental et industriel.

Au plan politique, le débat nucléaire qui renaissait depuis 2 ou 3 ans, a, dès la décision de principe du nouveau Gouvernement allemand de sortir du nucléaire, pris rapidement une dimension européenne en raison des accords commerciaux ou entre Etats concernant le retraitement du combustible et le retour dans leur pays d'origine des déchets correspondants. Mais au-delà, la décision allemande conduisait plusieurs pays européens à prendre position ; ainsi, le Premier ministre français réaffirmait le maintien de l'option nucléaire dans la politique énergétique nationale.

Au plan du marché de l'électricité, l'année écoulée a été également placée sous le signe de l'Europe. Après que la directive européenne sur l'électricité ait été adoptée en février 1997, puis transposée dans la plupart des pays de I'Union Européenne, le marché européen de l'électricité est véritablement né en 1999, avec une ouverture depuis le 19 février 1999 a minima aux clients industriels et, dans beaucoup de pays, généralisée à tous les consommateurs. Cette ouverture a eu des effets rapides, plus rapides qu'escomptés par certains, déclenchant une guerre des prix de l'électricité, en particulier en Allemagne. Dans ce contexte de compétition forte, de vastes mouvements de concentrations s'engagent : projet de fusion des deux conglomérats énergétiques allemands, Veba et Viag, qui, s'il aboutit, constituera le $n^{\circ} 2$ de l'électricité en Europe, compétition entre compagnies électriques pour acheter la part du Land de BadenWurtemberg dans le 4ème énergéticien allemand En $B W$, en Italie, vente par l'ENEL d'une part importante de son parc de production, etc...

II va de soi que la compétitivité du nucléaire est un facteur clef dans cette compétition européenne entre électriciens.

$\mathrm{Au}$ plan de l'environnement, les 15 Etats de l'Union Européenne se sont accordé en 1998 sur une répartition par pays de l'engagement collectif souscrit à Kyoto de réduire les émissions de gaz à effet de serre. Cet engagement européen de Kyoto, Mme de Palacio, nouvelle vice-présidente de la Commission Européenne, chargée en particulier de l'énergie, déclarait dernièrement impossible de le respecter sans la contribution de l'énergie nucléaire.

Les réflexions au sein de l'UE pour aboutir à une fiscalité harmonisée sur les consommations d'énergie ou les émissions de gaz à effet de serre se sont poursuivies sans aboutir pour l'instant, mais d'ores et déjà, plusieurs pays (Allemagne, Royaume-Uni, Italie) ont annoncé ou mis en œuvre en 1999 des programmes de taxation de l'énergie. La France, quant à elle, a décidé, d'élargir, à compter de 2001, le rôle de la taxe générale sur les activités polluantes (TGAP) à la prévention des pollutions globales, au premier rang desquelles l'émission des gaz à effet de serre.

Enfin, au plan industriel aussi, l'année écoulée a été placée sous le signe de l'Europe.

Tout d'abord, concernant les industriels de la construction de centrales électriques, la constitution d'ABB Alstom Power, intervenant un an après le rachat de Westinghouse Turbines par Siemens, "achève» le mouvement de concentration mondiale de ce secteur engagé à la fin des années 1980 ; désormais 4 acteurs mondiaux (ABB Alstom Power, General Electric, Siemens / Westinghouse, Mitsubishi) se partagent l'essentiel du marché mondial.

Alors que les commandes se raréfient, l'industrie nucléaire n'échappe pas à une concentration mondiale analogue : après la constitution de Westinghouse / BNFL l'an dernier, GE, Hitachi et Toshiba ont engagé la fusion de leurs activités dans le combustible nucléaire en une filiale commune.

Dans ce contexte, la recomposition du capital de Framatome, qui devrait faciliter l'établissement de partenariats industriels européens, revêt une importance majeure.

Dans un contexte aussi riche en actualités que rapide dans son évolution, nul doute que l'année à venir sera fertile dans la recomposition des paysages énergétiques et industriels européens.

Je me garderai de tout pronostic, mais j'ai la conviction que l'Europe sera encore plus fortement le cadre d'actions de tous les acteurs concernés par le nucléaire : pouvoirs publics, autorités de sûreté, électriciens, industriels,...

En tout cas, l'électronucléaire ne réussira que s'il sait recueillir la confiance du public, maintenir un haut niveau de sûreté et renforcer encore sa compétitivité. 